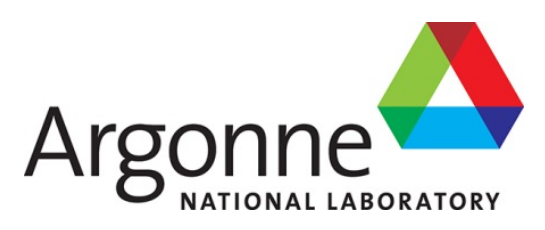

ANL/NSE-21/45

\title{
Initial use of Nek5000/Cardinal to improve closure models in Pronghorn
}

Nuclear Science and Engineering Division 


\section{About Argonne National Laboratory}

Argonne is a U.S. Department of Energy laboratory managed by UChicago Argonne, LLC under contract DE-AC02-06CH11357. The Laboratory's main facility is outside Chicago, at 9700 South Cass Avenue, Argonne, Illinois 60439. For information about Argonne and its pioneering science and technology programs, see www.anl.gov.

\section{DOCUMENT AVAILABLITY}

Online Access: U.S. Department of Energy (DOE) reports produced after 1991 and a growing number of pre-1991 documents are available free at OSTI.GOV (http://www.osti.gov/), a service of the US Dept. of Energy's Office of Scientific and Technical Information.

Reports not in digital format may be purchased by the public from the National Technical Information Service (NTIS):

U.S. Department of Commerce

National Technical Information Service

5301 Shawnee Rd

Alexandria, VA 22312

www.ntis.gov

Phone: (800) 553-NTIS (6847) or (703) 605-6000

Fax: (703) 605-6900

Email: orders@ntis.gov

Reports not in digital format are available to DOE and DOE contractors from the Office of Scientific and Technical Information (OSTI):

U.S. Department of Energy

Office of Scientifice and Technical Information

P.O. Box 62

Oak Ridge, TN 37831-0062

www.osti.gov

Phone: (865) 576-8401

Fax: (865) 576-5728

Email: reports@osti.gov

\section{Disclaimer}

This report was prepared as an account of work sponsored by an agency of the United States Government. Neither the United States Government nor any agency thereof, nor UChicago Argonne, LLC, nor any of their employees or officers, makes any warranty, express or implied, or assumes any legal liability or responsibility for the accuracy, completeness, or usefulness of any information, apparatus, product, or process disclosed, or represents that its use would not infringe privately owned rights. Reference herein to any specific commercial product, process, or service by trade name, trademark, manufacturer, or otherwise, does not necessarily constitute or imply its endorsement, recommendation, or favoring by the United States Government or any agency thereof. The views and opinions of document authors expressed herein do not necessarily state or reflect those of the United States Government or any agency thereof, Argonne National Laboratory, or UChicago Argonne, LLC. 
prepared by

Victor Coppo Leite ${ }^{1,2}$, Jun Fang ${ }^{1}$, David Reger ${ }^{2}$, Elia Merzari ${ }^{1,2}$, Haomin Yuan ${ }^{1}$, and Dillon Shaver ${ }^{1}$

${ }^{1}$ Nuclear Science and Engineering Division, Argonne National Laboratory

${ }^{2}$ Department of Nuclear Engineering, Pennsylvania State University

July 31,2021 


\section{Abstract}

Heat transfer coefficient closure models for pebble bed reactors are built using a data-driven approach by leveraging the capabilities of an Evolutionary Algorithm entitled Particle Swarm Optimization (PSO). In the present work, the Computational Fluid Dynamics code nekRS was used in order to collect the high-fidelity flow data for a core with 1,568 pebbles. To characterize the heat transfer, multiple concentric regions were considered to extract the physical quantities of interest, e.g./ the Reynolds number. The PSO algorithm is employed as part of an inverse problem targeting determine what are the coefficients for a Nusselt number correlation to match the collected data. Such correlation should follow any given format that is defined a priori. Finally, two correlations are proposed, one with an implicit dependence on the pebbles' wall temperatures and another expressed as a fully explicit correlation depending on the flow conditions and the position within the core. Anyway, given the generic nature of the proposed approach, correlations following different formats could be tested.

Preliminary results for the high-fidelity simulation of a fast MSR core are presented. The target Reynolds number is currently $20 \mathrm{~K}$, with the expectation that this will increase, pending the availability of further computational resources. These simulations will be used to inform lower fidelity models, including a coarse CFD turbulence model in Pronghorn. Additionally, they will serve as a reference for the RANS models in Nek5000/NekRS. 


\section{Contents}

Abstract ....................... i

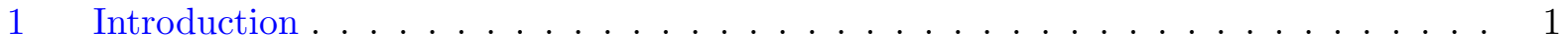

2 Heuristic algorithms for Pronghorn model development . . . . . . . . . . . . 1

2.1 Heat transfer coefficients from literature . . . . . . . . . . . . 2

2.2 Particle Swarm Optimization (PSO) $\ldots \ldots \ldots \ldots \ldots$

$2.3 \quad$ LES of a Pebble Bed Reactor . . . . . . . . . . . . . . . . . . 4

2.4 Data-driven approach with PSO to model the heat flux in Pebble Bed Reactors 5

$2.5 \quad$ Results and discussion . . . . . . . . . . . . . . . 7

3 LES model of a fast MSR core . . . . . . . . . . . . . . . . . . . . 10

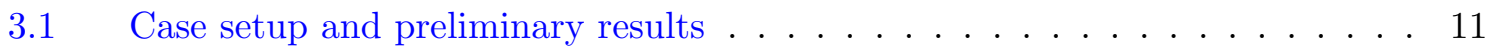

4 Conclusions and Continuing Work . . . . . . . . . . . . . . . . 13

Acknowledgments . . . . . . . . . . . . . . . . . . . . . 14

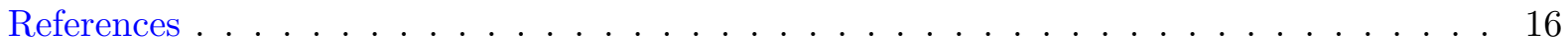




\section{List of Figures}

2.1 Particle Swarm Optimization algorithm pseudocode. . . . . . . . . . . . . 5

2.2 Time-averaged temperature field showing the concentric regions used to characterize the flow field, showing (a) a longitudinal cross-section view of the core with (b) a

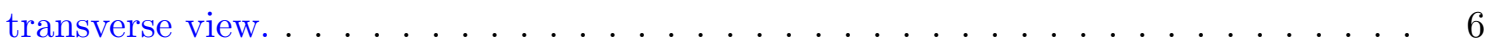

2.3 Fitness improvements considering both implicit and explicit formats provided by

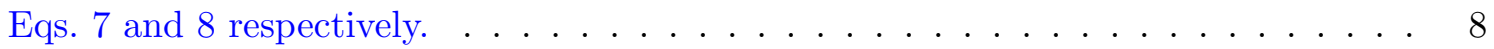

2.4 Spatial-average over the streamwise direction of the Nusselt number depending on the radial distance. . . . . . . . . . . . . . . . . . . . 9

2.5 Histogram of signed relative errors considering the concentric regions used to build the two PSO correlations. . . . . . . . . . . . . . . . . . . . 10

3.1 The structure and computational grid of the 3-D MSFR full core model. . . . . . . 12

3.2 A snapshot of developing turbulent velocity field in 3-D MSFR full-core model at Re

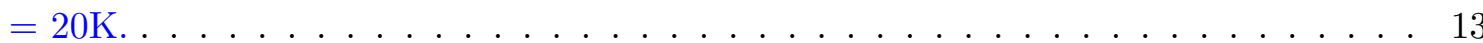

3.3 The steady-state solution fields of non-dimensional velocity (a) and turbulent kinetic energy (b) from the 2 -D RANS simulation at Re $=40 \mathrm{~K} \ldots \ldots \ldots \ldots$ 


\section{Introduction}

To develop the next generation of nuclear reactors will require accurate, fast-running, lower fidelity simulation tools designed for the express purpose of capturing the complicated physics associated with the proposed designs. These tools will need to be flexible enough to account for the wide variety of reactor types, while maintaining a high standard for accuracy. To accomplish this goal will require a modern approach to generating the underlying models used by these fast-running tools, relying on both traditional modeling techniques as well as data-driven techniques powered by novel Artificial Intelligence (AI) algorithms.

The focus of this work is on the use of high-fidelity CFD simulations to inform models for use in these fast-running tools. In particular, Large Eddy Simulations (LES) of flow through a pebble bed reactor, and flow through a Molten Salt Fast Reactor (MSFR) core are being performed with the NekRS and Nek5000 CFD tools. Data generated from these simulations is being used to inform model development for the lower fidelity tool, Pronghorn. The focus for the pebble bed reactor is in the evaluation of the use of AI to inform the development of heat transfer correlations. This has the potential to allow for the integration of large amounts of perspective data to enhance the ability of Pronghorn to accurately capture heat transfer phenomena in pebble beds. For the MSFR, more traditional methods are being pursued. Once completed, the LES data will be used to inform turbulence models integrated in the coarse CFD capability of Pronghorn. This will aid in the prediction of flow phenomena in the complex geometries associated with molten salt fueled reactor designs.

\section{Heuristic algorithms for Pronghorn model development}

Developing models that can accurately characterize the flow in randomly packed beds has been the subject of much research in the past few decades. Correlations for pressure drop and heat transfer were developed specifically for beds of randomly packed pebbles using experimental data. Application of these correlations is particularly interesting for Pebble-Bed Reactor (PBR) designs, a class of Generation IV of nuclear power reactors. These type of reactors features spherical fuel elements called pebbles, each of which consists of thousands of TRISO particles that are dispersed in a pyrolytic graphite material. The present work focuses on building heat transfer closure models for PBRs using high-fidelity data obtained via Computational Fluid Dynamic (CFD) simulation. We leverage the capabilities of an Evolutionary Algorithm entitled Particle Swarm Optimization (PSO) to address the inverse problem of determining the coefficients of any given correlation format such that the collected data is matched.

The goal of the present work is to develop tools that should couple closure models obtained from lower-length-scale simulations to engineering-length-scale ones. This is an existing demand in the development of Cardinal [1], a new platform for lower-length-scale simulation of pebble-bed cores. In the context of the present work, we employ the lower scale simulator NekRS to derive heat transfer formulations that might be supplied to engineering scale codes such as Pronghorn [2] and Mammoth [3]. 
Many correlations are available in the literature considering specifically randomly packed beds of pebbles. Refs. $[4,5]$ proposes correlations for the pressure drop in PBRs. The empirical correlation developed in Ref. [5] by the German Nuclear Safety Standards Commission (KTA) is particularly useful for nuclear applications. Furthermore, KTA also provides a heat transfer correlation that is also widely used [6].

These correlations are valuable tools for the development of PBRs as they allow calculating relevant design parameters, e.g. pressure drop and heat transfer capabilities, for the complex flow around fuel pebbles. Not only the above-mentioned KTA correlation, but also many others have been implemented in lower-fidelity codes such as Pronghorn [2] and PEBBED [7] to aid in analyzing PBRs.

The present work employs a PSO algorithm provided with high-fidelity simulation data to obtain the coefficients of two correlations with a priori defined form. Interestingly, this technique has already been successfully used to optimize a combinatorial problem of a Nuclear Reactor Reload Problem (NRRP) [8]. Large Eddy Simulation (LES) of a core with 1,568 pebbles have been carried out using the Computational Fluid Dynamics (CFD) code NekRS [9] to collect high-fidelity distributions of velocity and temperature. Multiple concentric regions were considered to extract the physical quantities of interest to characterize the heat transfer, e.g. the local Reynolds number. Two correlation formats have been considered. The first featuring an implicit dependence on the pebbles' wall temperatures and the second is expressed as a fully explicit formulation depending on the flow conditions and the spatial position within the core. We show that the lower-length-scale simulator (NekRS) aided with the PSO algorithm can provide correlations in an off-line fashion which can be used in engineering scale tools. Another possible option that may be explored in the future is to concurrently provide dynamic closures from the former to the latter. Finally, the strategy proposed here is completely data-driven. Hence, in principle it could be employed for any type of correlation of interest.

Here we investigate the capability of a heuristic algorithm entitled Particle Swarm Optimization to retrieve the coefficients of a given Nusselt number correlation. PSO is classified as an Evolutionary Algorithm that intends to mimic swarm intelligence. This technique, along with others such as Neural Networks and fuzzy logic, are considered to be Computational Intelligence (CI) algorithms, a branch of the broader field of Artificial Intelligence (AI) [10].

There are two main advantages in using PSO instead of other techniques to carry out the present work. First, it has fewer parameters to adjust and the values for these parameters have been widely discussed in the literature [11]. Second, it is well suited to problems featuring continuum variables, which is the case here since the set of parameters that we are looking for are assumed to be real numbers. The PSO algorithm is described in the next section. Later, Section 2.4 describes how this algorithm has been employed for the task of building a new Nusselt number correlation.

\subsection{Heat transfer coefficients from literature}

Engineering scale codes devoted to PBR analysis often use heat transfer coefficient correlations available in the literature valid for beds of spherical pebbles. Pronghorn [2] is able to use the 
correlation provided by the German Nuclear Safety Standards Commission (KTA) [5], Eq. (1).

$$
N u=1.27 \frac{\operatorname{Pr}^{1 / 3} \operatorname{Re}^{0.36}}{\varepsilon^{1.18}}+0.033 \frac{\operatorname{Pr}^{0.5} \operatorname{Re}^{0.86}}{\varepsilon^{1.07}}
$$

This correlation is valid for $100 \leq R e \leq 10^{5}$, and a bed height greater than four times the pebble diameter. It uses local values for the Reynolds and Prandtl numbers, meaning that the definition of $R e=\rho \bar{U} D_{p e b} / \mu$ should be applied. Here, $\bar{U}$ is the intrinsic phase velocity.

It should be noted that special attention is given to the outer ring where the coolant is in contact with the solid walls of the core. Heat transfer occurs through the thermal boundary layer, which is generally much smaller than the pebble diameter. For turbulent regimes, heat removal is carried out mainly by convection in the outer ring provided the fact that the solid thermal conductivity was found to not have a significant impact on the wall heat transfer coefficient [12]. For this region, Pronghorn should employ Eq. (2), a correlation experimentally verified by Achenbach in Ref. [13] which is valid within $50 \leq R e \leq 2 \times 10^{4}$.

$$
N u=\left(1-\frac{D_{p e b}}{D_{b e d}}\right) R e^{0.61} R e^{1 / 3}
$$

\subsection{Particle Swarm Optimization (PSO)}

Kennedy and Eberhart first proposed the Particle Swarm Optimization algorithm in 1995 [14]. This method features a metaheuristic algorithm based on the concept of swarm intelligence and it is capable of solving complex optimization problems with multiple variables and objectives.

The swarm individuals are termed as particles. The key idea of the algorithm is that each particle balances its own (local) knowledge and the swarm (global) knowledge when exploiting a continuous domain. The algorithm mimics the social behavior present in swarms, where all the individuals can share information among themselves. In nature, for instance, this capability helps the swarm to find a position in terms of latitude and longitude where the survival conditions are optimized, e.g. maximizing the availability of food and minimizing the threat of predators. Considerable effort was spent by previous authors [14] to abstract the social behavior often observed in swarms of animals, e.g. birds or fish, to develop the PSO algorithm.

In essence, an optimization problem seeks to either minimize or maximize a function $f(X)$, which in turn features a search problem of the variable $X$ in its domain. In the PSO algorithm, $X$ is termed as position variable, in reference to the search for latitude and longitude as in the above mentioned example, whereas $f(X)$ is often called the fitness function. Considering that the problem being addressed has $n$ variables, each particle of the swarm, $i$, spans a solution vector $X_{i}^{t}=\left(x_{i}, 1, x_{i}, 2, \ldots, x_{i}, n\right)^{T}$ throughout many iterations until a given criteria is met.

Each one of all $P$ particles in a swarm represents a position vector $X$ which is updated on every iteration $t$ with a velocity vector of the same format $V_{i}^{t}=\left(v_{i}, 1, v_{i}, 2, \ldots, v_{i}, n\right)^{T}$. Equations $(3$ and 4$)$ 
show how these two variables are updated.

$$
\begin{gathered}
V_{i}^{t+1}=w \cdot V_{i}^{t}+c_{1} \cdot r_{1} \cdot\left(p_{\text {best }_{i}}-X_{i}^{t}\right)+c_{2} \cdot r_{2} \cdot\left(g_{\text {best }}-X_{i}^{t}\right) \\
X_{i}^{t+1}=X_{i}^{t}+V_{i}^{t+1}
\end{gathered}
$$

A brief description of each of these equations is provided below. For the velocity, Eq. (3):

- $w \cdot V_{i}^{t}$ - is the inertial term and it accounts for the influence of a particle's previous motion on a current one. The parameter $w$ is the inertia weight and should have a constant and positive value. This parameter balances the global search (when higher values are used) and the local search (when lower values are used instead). Thus, adjusting the importance of this term may favor either a local or a global search for an optimum solution.

- $c_{1} \cdot r_{1} \cdot\left(p_{\text {best }_{i}}-X_{i}^{t}\right)$ - is the own-cognition term and it represents a particle's individual knowledge about the domain being explored. This term accounts for the influence of the best solution ever found by a single particle $p_{\text {best }}$ when the particle is updating its solution $X_{i}^{t}$ to the next iteration.

- $c_{2} \cdot r_{2} \cdot\left(g_{\text {best }}-X_{i}^{t}\right)$ - is the swarm-cognition term and it represents the knowledge of all the individuals of the swarm about the domain being explored. This term plays a similar role as the previous one, but it accounts for the influence of the best solution ever found by any of the particles, $g_{\text {best }}$. Both $c_{1}$ and $c_{2}$ are social-cognition parameters and they must have constant and positive values. However, $r_{1}$ and $r_{2}$ are random values following a uniform distribution.

For the position, Eq. (4):

- The previous position of all particles $X_{i}^{t}$ is updated to a new one $X_{i}^{t+1}$ after the velocity vector is updated considering the three terms described above.

It should be noted that although there are different versions of the PSO algorithm available, Eqs. (3 and 4) feature the inertial formulation proposed by Shi and Eberhart [15]. Finally, Figure 2.1 provides the pseudocode for the PSO algorithm.

\subsection{LES of a Pebble Bed Reactor}

Large Eddy Simulation is performed using NekRS as it is able to leverage modern GPU computing capabilities to perform large simulations. NekRS is a new GPU-oriented version of Nek5000 [16], an open-source CFD code developed at Argonne National Laboratory. It can link to Nek5000 and leverage both of its pre- and post-processing utilities. Details of NekRS performance and capabilities in nuclear applications can be found in [17]. 


\section{Initialization}

1.1. For each particle $\mathrm{i}$ in a swarm population size $\mathrm{P}$ :

1.1.1. Initialize $X_{i}$ randomly

1.1.2. Initialize $V_{i}$ randomly

1.1.3. Evaluate the fitness $f\left(X_{i}\right)$

1.1.4. Initialize pbest $_{i}$ with a copy of $X_{i}$

1.2. Initialize gbest with a copy of $X_{i}$ with the best fitness

2. Repeat until a stopping criterion is satisfied:

2.1. For each particle i:

2.1.1. Update $V_{i}^{t}$ and $X_{i}^{t}$ according to Eqs. (1) and (2)

2.1.2. Evaluate the fitness $f\left(X_{i}^{t}\right)$

2.1.3. pbest $_{i}-X_{i}^{t}$ if $f\left(\right.$ pbest $\left._{i}\right)<f\left(X_{i}^{t}\right)$

2.1.4. gbest $X_{i}^{t}$ if $f($ gbest $)<f\left(X_{i}^{t}\right)$

Figure 2.1: Particle Swarm Optimization algorithm pseudocode.

The present work considered one case with 1,568 pebbles targeting a porosity of $\varepsilon=0.4$. A hexahedral-element mesh has been developed for this case based on a tessellation of a Voronoi diagram [18]. It should be noted that this case models the contact points between pebbles by including a small gap. This configuration is part of a geometry simplification to avoid numerical issues caused by the large deformations imposed on the mesh caused by these regions.

In the considered case, the flow is at $R e_{b}=20,000$, a Prandtl of $\operatorname{Pr}=0.8$, and a unity heat flux, i.e. $q^{\prime \prime}=1$, is applied at the surface of all pebbles. Furthermore, the boundaries of the core have an insulated boundary condition for the temperature field. It should be noted that the Reynolds number definition considered here is $R e_{b}=\rho v D_{p e b} / \mu$. In this definition, $\rho$ is the density, $\mu$ is the dynamic viscosity, $D_{\text {peb }}$ is the diameter of the pebbles, and $v$ is the Darcy velocity. The Darcy velocity, also known as superficial velocity or extrinsic velocity [2], can be related to the phasic velocity, $u$, as $v=u \varepsilon$, and represents the fluid velocity averaged over the entire medium (both solid and fluid). Finally, as it will be shown, sufficient time-averaging has been considered to ensure statistically converged results.

\subsection{Data-driven approach with PSO to model the heat flux in Pebble Bed Reactors}

High-fidelity data provided by NekRS is averaged over several smaller subdomains within the pebble bed where the physical quantities of interest have been computed. These subdomains break up the full pebble bed core into several concentric annular regions of uniform thickness along the axis of the core. an example of which is shown in Figure 2.2.

It should be noted that Figure 2.2 is merely representative as the quantities of rings and layers 


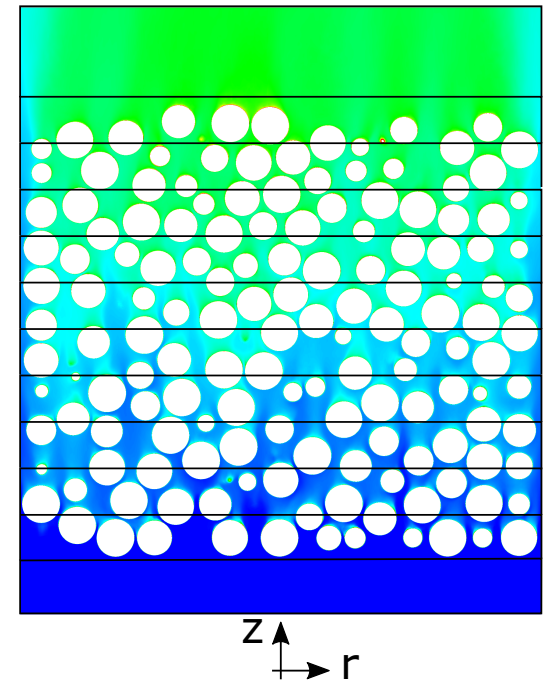

(a)

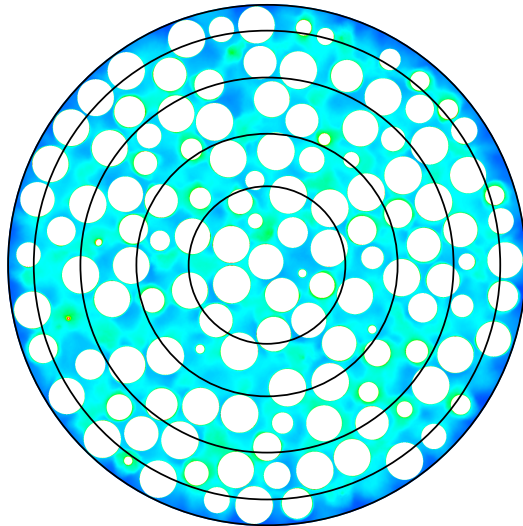

(b)

Figure 2.2: Time-averaged temperature field showing the concentric regions used to characterize the flow field, showing (a) a longitudinal cross-section view of the core with (b) a transverse view.

are arbitrarily represented. In the actual setup, 5 layers have been considered in the streamwise direction where each is subdivided into 4 concentric regions. These regions have been defined such that each one of them contains at least one pebble in the radial direction and roughly two layers of pebbles in the streamwise direction.

The bulk Nusselt number is calculated and compared to available correlations from the literature. A similar procedure to the one described in [19] is employed to obtain the average Nusselt numbers in each of the regions. First, the time-average temperature fields for the fluid $\bar{T}_{b}$ and for the heated walls $\bar{T}_{w}$ are calculated. The local-average Nusselt number is then calculated from:

$$
\bar{N} u=\frac{q^{\prime \prime}}{\bar{T}_{w}-\bar{T}_{b}} \frac{D_{h}}{k}
$$

Where $k$ is the conductivity and the standard hydraulic diameter in a porous medium is defined as [2]:

$$
D_{h}=\frac{4 \varepsilon}{6(1-\varepsilon)} D_{p e b}
$$

Two correlation formats have been considered in order to employ the PSO algorithm to model the Nusselt number. These were and implicit correlation, Eq. (7), which depends on the heated wall temperature $T_{w}$, and an explicit correlation, Eq. (8), with a spatial dependence of the radial distance $r$ within the core. 


$$
\begin{gathered}
N u=A \frac{\operatorname{Pr}^{B} \operatorname{Re}^{C}\left(T_{w}-T_{b}\right)^{D}}{\varepsilon^{E}} \\
N u=A \operatorname{Re}^{B} \operatorname{Pr}^{C}+D\left(1-\frac{r}{R_{\text {bed }}}\right)^{E} \operatorname{Re}^{F} \operatorname{Pr}{ }^{G}
\end{gathered}
$$

Each proposed form attempts to account for the variability in the local porosity through two different mechanisms. The implicit form, Eq. (7) includes the local porosity directly as a parameter, whereas the explicit form Eq. (8) includes a radial position dependence. These correlations are tailored to be employed using local values for both Reynolds and Prandtl numbers. This way, the Reynolds number must be computed according to $R e=\rho \bar{U} D_{p e b} / \mu$ rather than the $R e_{b}$ definition provided in Section 2.3. Here, $\bar{U}$ represents the fluid velocity averaged over only the fluid domain in a given region, and it is referred to as the intrinsic phase velocity.

From the PSO perspective, the set of coefficients provided by Eqs. (7 and 8) features the position variable $X$ to be determined in order to minimize the fitness function $f(X)$. That is, the position variable of a particle $i$ at a certain iteration $t$ of the PSO algorithm should be written as $X_{i}^{t}=\left(A_{i}^{t}, B_{i}^{t}, C_{i}^{t}, D_{i}^{t}, E_{i}^{t}\right)^{T}$ considering Eq. (7) and $X_{i}^{t}=\left(A_{i}^{t}, B_{i}^{t}, C_{i}^{t}, D_{i}^{t}, E_{i}^{t}, F_{i}^{t}, G_{i}^{t}\right)^{T}$ for Eq. (8). Furthermore, the fitness function should be developed aiming to reflect some engineering knowledge of the problem being addressed in order to avoid the algorithm converging to a nonsense solution. For this reason, defining this function is a key aspect not only in the PSO algorithm but also in any other Evolutionary Algorithm [20]. The function used in the present work is

$$
f(X)=\sum_{j=1}^{N}\left|N u_{j}-N u_{j}^{*}\right|
$$

The index $j$ indicates the region from Figure 2.2 and $N$ represents the total of regions being evaluated by the PSO algorithm. For the present work, $N=16$ given that the first layer of concentric regions is not being evaluated due to entrance effects. Continuing, $N \bar{N} u_{j}$ represents the Nusselt number calculated from the LES using Eq. (5) whereas $N u_{j}^{*}$ is the Nusselt number provided by a particle in the swarm. These two quantities are calculated based on local values of region $j$.

Finally, the PSO simulations were performed by setting the parameters to $P=600$ particles, an inertial weight of $w=0.01$. Additionally, $c_{1}=1.8$ and $c_{2}=2.2$ are chosen such that their sum is 4.0 and a global attractor is formed, which helps the convergence process [21].

\subsection{Results and discussion}

The time-averaged Nusselt numbers is computed according to Eq. (5) for various regions of the core, Figure 2.2. After reaching a statistically steady-state, the 1,568 pebbles case was run for six more flow-through time units to collect statistics such that fully converged results were obtained. 
Then, the PSO algorithm is employed in order to determine the coefficients of the two proposed correlations provided by Eqs. (7 and 8) such that it best fits into the obtained local Nusselt numbers. The convergence of the fitness function throughout 200 iterations for both implicit and explicit formats is shown in Figure 2.3.

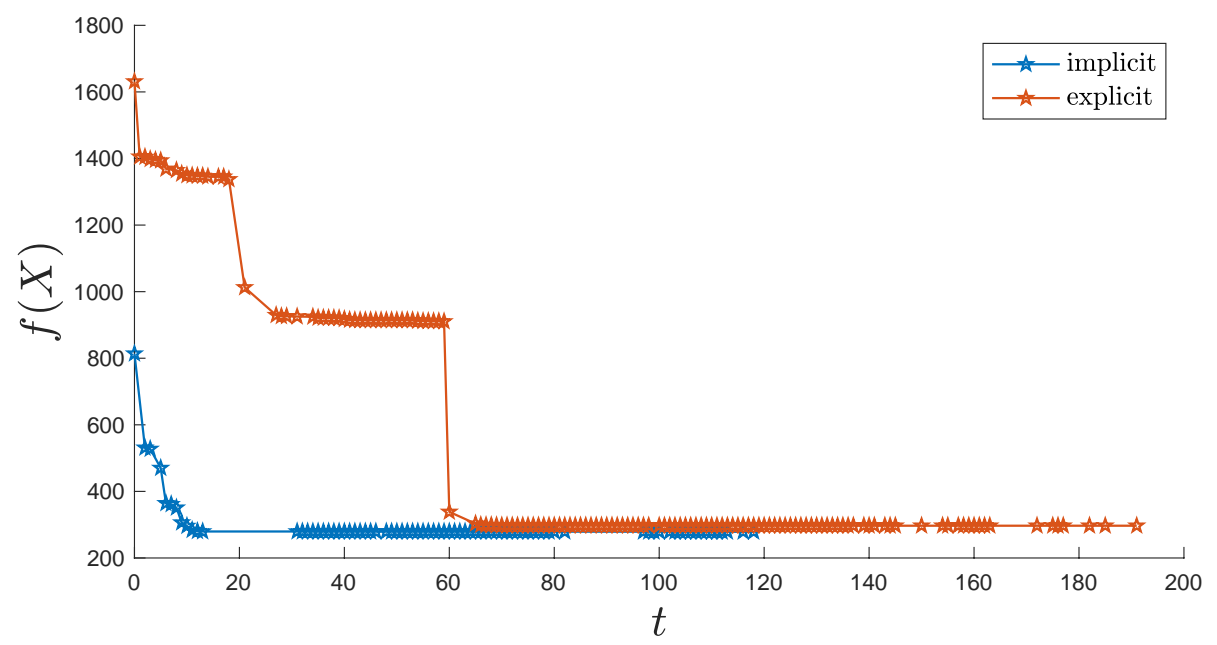

Figure 2.3: Fitness improvements considering both implicit and explicit formats provided by Eqs. 7 and 8 respectively.

It is clear from Figure 2.3 that both explicit and implicit formats converge to similar values. However, it should be noted that the implicit format has a clear advantage as it features a faster convergence than the explicit one. A potential explanation for that is the fact that Eq. (7) depends on fewer parameters and it also provides more physical information about the problem being addressed, which in turn may help to find an optimum solution. Still, the implicit format imposes a drawback due to its dependence on $T_{w}$, which requires an iterative scheme if this format is used in an engineering scale simulator such as Pronghorn.

The parameter values obtained by the PSO algorithm are provided in Eqs. (10 and 11) for the implicit and the explicit formats respectively.

$$
\begin{gathered}
N u=6.95 \frac{\operatorname{Pr}^{0.41} \operatorname{Re}^{0.63}\left(T_{w}-T_{b}\right)^{-1.31}}{\varepsilon^{1.15}} \\
N u=5.74 \operatorname{Re}^{0.52} \operatorname{Pr}^{1.85}-0.12\left(1-\frac{r}{R_{b e d}}\right)^{-2.2} \operatorname{Re}^{0.19} \operatorname{Pr}^{1.35}
\end{gathered}
$$

Figure 2.4 presents the Nusselt number spatially-averaged over the streamwise direction as a function of the radial distance. In this plot, the Pronghorn predictions were obtained by applying the KTA correlation, Eq. (1), for the inner regions whereas the outer region results from the Achenbach correlation, Eq. (2). 


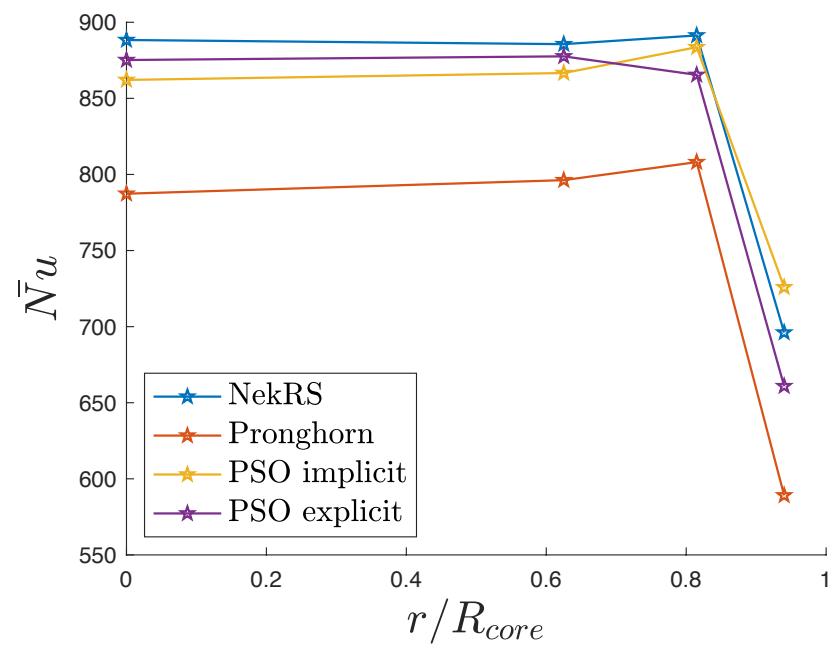

Figure 2.4: Spatial-average over the streamwise direction of the Nusselt number depending on the radial distance.

Figure 2.5 shows the distributions of the signed relative errors between the NekRS results and the PSO correlations considering the 16 concentric regions on which these two correlations were developed. In general, the errors are centered at $\epsilon=0.0 \%$ for both implicit and explicit formats. Thus, it can be inferred from these histograms that the PSO correlations are in good agreement with the calculated values from the CFD simulation.

Interestingly, reasonable agreement is observed between the results obtained via LES using NekRS and the predictions made by employing the correlations available in Pronghorn. This was somewhat unexpected as the NekRS model does not account for pebble-to-pebble contact. However, as expected the PSO-derived correlations show much better agreement. Table 2.1 reports the maximum relative errors between the correlations shown in Figure 2.4 and the NekRS results. Errors are given in percentages of the NekRS results.

Table 2.1: Relative errors errors between the coefficient correlations for the Nusselt number and the results obtained using NekRS.

\begin{tabular}{cl}
\hline Correlation & $\epsilon(\%)$ \\
\hline Eqs. (1 and 2) (Pronghorn) & 15.3 \\
PSO (implicit) & 4.3 \\
PSO (explicit) & 5.0 \\
\hline
\end{tabular}




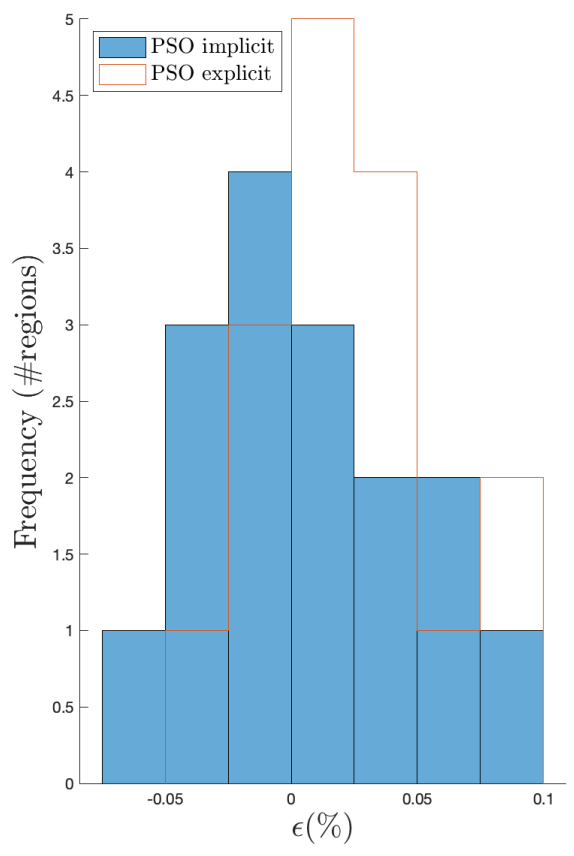

Figure 2.5: Histogram of signed relative errors considering the concentric regions used to build the two PSO correlations.

\section{LES model of a fast MSR core}

In addition to the development of data-driven closure models for pebble bed reactors, an exploratory investigation is also conducted in this modeling and simulation campaign for the molten salt reactor (MSR). The related efforts are beneficial to the Pronghorn development, especially on the turbulence modeling for MSR related applications. The Molten Salt Reactor (MSR) stands out as a promising candidate among advanced nuclear reactor concepts with its improved passive safety characteristics and high thermal efficiency. In MSRs, fissile material (fuel) is dissolved in a molten salt, which is typically chloride or fluoride based. This represents a significant paradigm shift compared to traditional light-water or other advanced reactors which use solid fuel rods. Among the MSRs, there are fast spectrum MSRs with flowing fuel salt through open cores, such as the MSFR concept designed as part of the Euratom EVOL (Evaluation and Viability of Liquid Fuel Fast Reactor Systems) project. This concept has been selected as the main reference in this study due to its large negative temperature and void reactivity coefficient. One primary feature of the MSFR is the absence of solid moderators or flow channels inside the core compared to regular thermal-neutron MSR designs. The core cavity walls thus serve as the major constraint to achieve specific internal flow distribution. Since the fuel in an MSFR is already in a liquid state, the melting point of the fuel is no longer a limiting factor in the reactor design, completely eliminating a major safety concern. However, this brings its own set of design challenges. For example, one major challenge that comes with the MSFR design is the configuration of multiple inlet channels entering the bottom of the core 
at relatively high velocities. It is found out that such configuration is prone to generate large-scale re-circulation zones that could potentially cause temporal and spatial instabilities in the reactor power distribution and subsequent excessive temperatures. Other major design challenges associated with the MSFR include: (i) the need of accurately tracking the delayed neutron precursors, (ii) the lack of suitable thermal-hydraulic closures for high-Prandtl fluid like the chloride or fluoride based molten salts, and etc.

To address the related design challenges, high-quality databases are demanded to better model the fundamental thermal-hydraulic phenomena in a fast MSR system. Because of the potential high costs associated with building relevant experimental facilities, the CFD approach is becoming increasingly attractive to produce the needed reference data, especially the LES method given the necessary computational resources. In this study, we perform the LES simulations of a 3-D MSFR full core model to shed light on the complex thermal-hydraulic phenomena in the MSFR core cavity. The case setups and preliminary results are detailed in the following sections. The present investigation lays a solid foundation as we are leveraging the high-fidelity CFD capabilities to improve the prediction accuracy of lower-fidelity nuclear reactor design code, such as Pronghorn. Specifically, results from the LES model will be used to benchmark turbulence models implemented using the coarse CFD capability of Pronghorn.

\subsection{Case setup and preliminary results}

The specific reactor model considered herein is based upon the MSFR concept developed under the Euratom EVOL project [22, 23]. The reference MSFR is a $3000 \mathrm{MW}$ fast-spectrum reactor with three different circuits: the fuel circuit, the intermediate circuit and the power conversion circuit. In the fuel circuit, there are 16 groups of pumps and heat exchangers around the core. A representative full core model is created for the MSFR core cavity using the Nek5000. Leveraging the existing MSFR studies published in the literature, the "Geometry II" investigated by Rouch et al. [23] is selected as the reference geometry for our CFD simulations. The related investigations will first focus on the velocity distributions in the core cavity, which is used to demonstrate the applicability of NEAMS CFD code (i.e., Nek5000) in MSFR related thermal-fluid problems. The next step is to include more physics in the MSFR simulations, such as the heating and the tracking of delayed neutron precursors, which will be investigated in the near future. Since a full-core wall-resolved LES of the MSFR core requires a considerable amount of computing power ${ }^{1}$, a demonstrative full core LES case with the coarsened mesh and relatively low-polynomial order is conducted with the Nek5000, which is used to prove the feasibility of a high-fidelity CFD model of the 3-D MSFR full-core.

The MSFR core has a height of $1.6 \mathrm{~m}$ along the centerline, and a height of $2.65 \mathrm{~m}$ in the peripheral region. The reactor radius ranges from 1.05 to $1.53 \mathrm{~m}$. The peripheral wall is a curved surface, which resembles the shape of an hourglass. The geometric model and mesh are generated using the open source meshing software, GMSH [24]. The entire model consists of the core cavity

\footnotetext{
${ }^{1}$ A quick estimation shows that it requires over 3 million spectral elements, or 1 billion grid points to perform the wall-resolved LES of MSFR full core at Re $=250,000$ (a quarter of the expected Reynolds number under the normal operating condition).
} 
region and the inlet and outlet channels. Our scoping study shows that the curved cavity walls and the bent inlet/outlet elbows are essential in producing a relatively uniform velocity distribution inside the core cavity. Moreover, the inlet channel is extruded accordingly to have a more developed inflow condition when the molten salt enters the core, while the extrusion of outlet helps prevent the back-flow issue. As shown in Figure 3.1, sixteen external loops are considered in the 3-D model around the MSFR core. Note that the ex-core components in the primary loop, such as the pumps and heat exchangers, are not modeled in this work. Future work may consider modeling these components via porous media models, especially for the heat exchanger regions. Some preliminary results from the 3-D MSFR full core simulations is discussed in the next section.

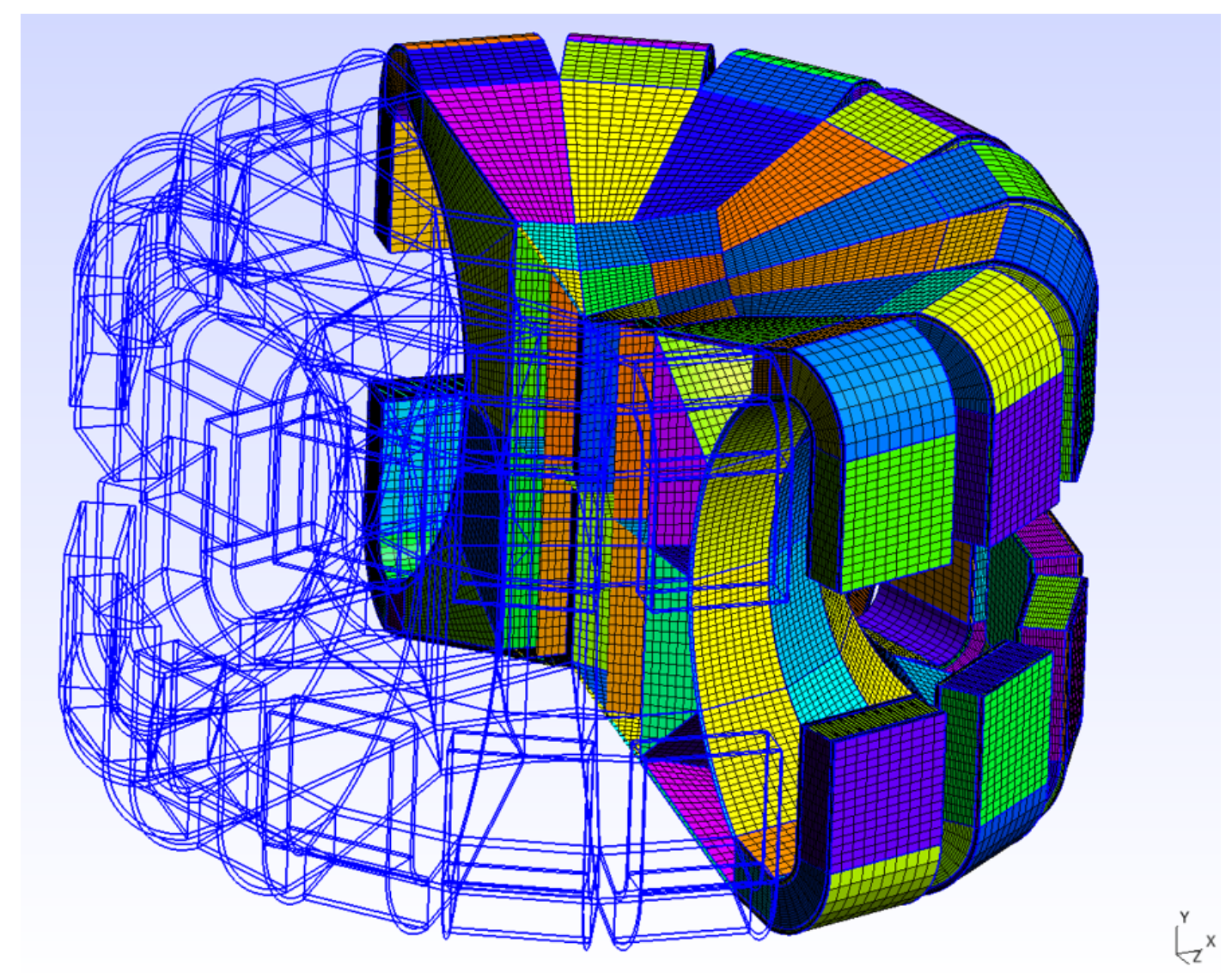

Figure 3.1: The structure and computational grid of the 3-D MSFR full core model.

As mentioned earlier, a demonstrative full-core LES case is simulated on a coarsened mesh of 660,000 elements at a polynomial order of 5. The corresponding Reynolds number is about 20,000 based on the mean velocity through minimum core diameter. A turbulent outflow treatment is applied on the outflow faces to avoid the back-flow issue. As shown in Figure 3.2, strong turbulence is being developed at the core bottom where the molten salt is injected into the core cavity. The regions of large velocity fluctuation correspond well to the high TKE spots revealed by the RANS simulations 
shown in Figure 3.3. The LES simulation is likely under-resolved due to the limited computational resources currently available, because a wall-resolved LES would require a considerable amount of CPU-hours to carry out on leadership class supercomputers. Having said that, it is clear that a 3-D MSFR full core LES is feasible. The related simulations are an important stepping stone as we are trying to leverage high-quality CFD calculations to enhance the prediction accuracy of coarse-grid CFD and/or reactor system codes used in actual MSFR design efforts.

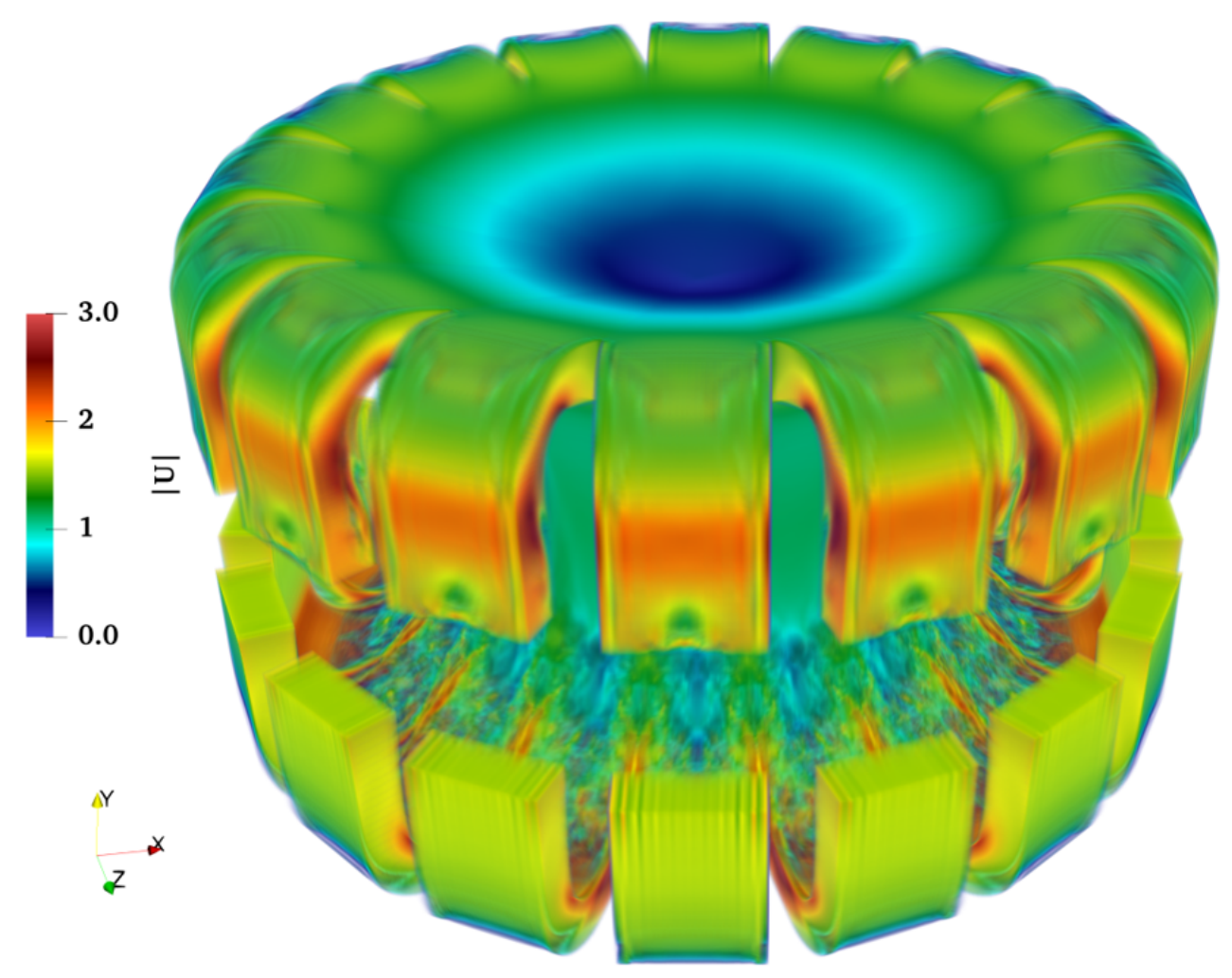

Figure 3.2: A snapshot of developing turbulent velocity field in 3-D MSFR full-core model at Re = $20 \mathrm{~K}$.

\section{Conclusions and Continuing Work}

The PSO algorithm proved to be capable of retrieving the coefficients of correlations considering different formats, hence featuring an effective data-driven approach to obtain closure models. Besides that, the correlations available in Pronghorn also proved to be, to some extent, in agreement with NekRS results. For future work, other algorithms may be tested besides the PSO, e.g. the Genetic 

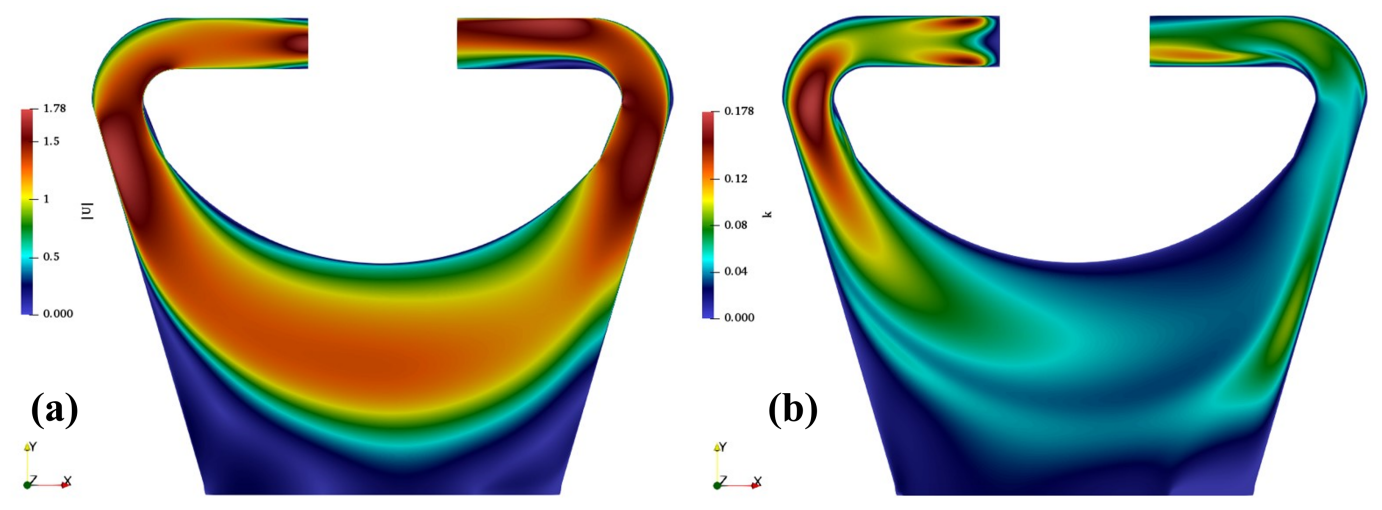

Figure 3.3: The steady-state solution fields of non-dimensional velocity (a) and turbulent kinetic energy (b) from the 2-D RANS simulation at $\mathrm{Re}=40 \mathrm{~K}$.

Algorithm (GA) and Neural Networks. Additionally, more conditions should be considered when obtaining the correlations by varying not only the Reynolds or the Prandtl numbers, but also and the porosity. Finally, given the generic nature of the present strategy, other coefficient correlations will be also investigated. For instance, in [25] a drag coefficient correlation was manually adjusted such that Pronghorn results matched NekRS. This way, the capabilities of heuristic algorithms may be useful to address this and other kinds of problems.

Initial results for the practical use of LES for performing high-fidelity simulations of turbulent flow and heat transfer in a full MSFR core have been shown. The proposed simulations will be continued on the available computational resources at moderate Reynolds numbers to provide a benchmark for the coarse CFD models implemented in Pronghorn. We expect to be able to begin providing converged results for $\mathrm{Re}=20,000$ by the end of FY21. Additionally, higher Reynolds numbers will be simulated as leadership class resources become available into FY22.

\section{Acknowledgments}

Argonne National Laboratory's work was supported by the U.S. Department of Energy, Office of Nuclear Energy, Nuclear Energy Advanced Modeling and Simulation (NEAMS) program, under contract DE-AC02-06CH11357. 


\section{References}

[1] E. Merzari, H. Yuan, M. Min, D. Shaver, R. Rahaman, P. Shriwise, P. Romano, A. Talamo, Y.-H. Lan, D. Gaston, R. Martineau, P. Fischer, and Y. Hassan, "Cardinal: A lower-lengthscale multiphysics simulator for pebble-bed reactors," Nuclear Technology, vol. 207, no. 7, pp. 1118-1141, 2021.

[2] A. Novak, D. Andrs, J. Kelly, R. Slaybaugh, R. Martineau, S. Schunert, R. W. Carlsen, and P. Balestra, "Pronghorn theory manual," 52020.

[3] J. Ortensi, Y. Wang, F. Gleicher, S. Schunert, D. Gaston, C. Permann, and R. C. Martineau, "Mammoth, version 00," 62017.

[4] I. Kececioglu and Y. Jiang, "Flow Through Porous Media of Packed Spheres Saturated With Water," Journal of Fluids Engineering, vol. 116, pp. 164-170, 031994.

[5] "Reactor core design of high-temperature gas-cooled reactors part 3: Loss of pressure through friction in pebble bed cores," Tech. Rep. KTA 3102.3, 1981.

[6] "Reactor core design of high-temperature gas-cooled reactors part 3: Heat transfer in spherical fuel elements," Tech. Rep. KTA 3102.2, 1983.

[7] H. D. Gougar, A. M. Ougouag, and W. K. Terry, "Advanced core design and fuel management for pebble-bed reactors," 102004.

[8] A. A. de Moura Meneses, M. D. Machado, and R. Schirru, "Particle swarm optimization applied to the nuclear reload problem of a pressurized water reactor," Progress in Nuclear Energy, vol. 51, no. 2, pp. 319-326, 2009.

[9] P. Fischer, S. Kerkemeier, M. Min, Y. Lan, M. Phillips, T. Rathnayake, E. Merzari, A. Tomboulides, A. Karakus, N. Chalmers, and T. Warburton, "NekRS, a gpu-accelerated spectral element navier-stokes solver," CoRR, vol. abs/2104.05829, 2021.

[10] K. Wang, Agile Manufacturing: The 21st Century Competitive Strategy, ch. Computational Intelligence in Agile Manufacturing Engineering. Elsevier, 2001.

[11] S. Sarkar, A. Roy, and B. S. Purkayastha, "Application of particle swarm optimization in data clustering: A survey," International Journal of Computer Applications, vol. 65, pp. 38-46, 2013. 
[12] S. Yagi and N. Wakao, "Heat and mass transfer from wall to fluid in packed beds," AIChE Journal, vol. 5, no. 1, pp. 79-85, 1959.

[13] E. Achenbach, "Heat and flow characteristics of packed beds," Experimental Thermal and Fluid Science, vol. 10, no. 1, pp. 17-27, 1995.

[14] J. Kennedy and R. Eberhart, "Particle swarm optimization," in Proceedings of ICNN'95 International Conference on Neural Networks, vol. 4, pp. 1942-1948 vol.4, 1995.

[15] Y. Shi and R. Eberhart, "A modified particle swarm optimizer," in 1998 IEEE International Conference on Evolutionary Computation Proceedings. IEEE World Congress on Computational Intelligence (Cat. No.98TH8360), pp. 69-73, 1998.

[16] Nek5000 Version 19.0. December, 2019. Argonne National Laboratory, Argonne, Illinois. Available: https://nek5000.mcs.anl.gov.

[17] E. Merzari, P. Fischer, M. Min, S. Kerkemeier, A. Obabko, D. Shaver, H. Yuan, Y. Yu, J. Martinez, L. Brockmeyer, L. Fick, G. Busco, A. Yildiz, and Y. Hassan, "Toward exascale: Overview of large eddy simulations and direct numerical simulations of nuclear reactor flows with the spectral element method in nek5000," Nuclear Technology, vol. 206, no. 9, pp. 1308-1324, 2020 .

[18] J. M. Kang, Voronoi Diagram, pp. 1232-1235. Boston, MA: Springer US, 2008.

[19] D. R. Shaver, L. B. Carasik, E. Merzari, N. Salpeter, and E. Blandford, "Calculation of friction factors and nusselt numbers for twisted elliptical tube heat exchangers using nek5000," $J$. Fluids Eng., 2019.

[20] B. S. G. de Almeida and V. C. Leite, "Particle swarm optimization: A powerful technique for solving engineering problems," in Swarm Intelligence (J. D. Ser, E. Villar, and E. Osaba, eds.), ch. 3, Rijeka: IntechOpen, 2019.

[21] I. C. Trelea, "The particle swarm optimization algorithm: convergence analysis and parameter selection," Information Processing Letters, vol. 85, no. 6, pp. 317-325, 2003.

[22] B. Yamaji, A. Aszódi, M. Kovács, and G. Csom, "Thermal-hydraulic analyses and experimental modelling of MSFR," Annals of Nuclear Energy, vol. 64, pp. 457-471, 2014.

[23] H. Rouch, O. Geoffroy, P. Rubiolo, A. Laureau, M. Brovchenko, D. Heuer, and E. Merle-Lucotte, "Preliminary thermal-hydraulic core design of the Molten Salt Fast Reactor (MSFR)," Annals of Nuclear Energy, vol. 64, pp. 449-456, 2014.

[24] C. Geuzaine and J.-F. Remacle, "Gmsh: A 3-D finite element mesh generator with built-in preand post-processing facilities," International Journal for Numerical Methods in Engineering, vol. 79, pp. 1309-1331, sep 2009.

[25] D. Reger, E. Merzari, P. Balestra, S. Schunert, and Y. A. Hassan, "Comparison of pebble bed velocity profiles between high-fidelity and intermediate-fidelity codes," in Proceedings of the ICONE 28 International Conference on Nuclear Engineering, no. ICONE28-65759, 2021. 


\section{Argonne}

Nuclear Science and Engineering Division

Argonne National Laboratory

9700 South Cass Avenue, Bldg. 208

Argonne, IL 60439

www.anl.gov 